\title{
Charmonium Mass Spectrum with Spin-Dependent Interaction in Momentum-Helicity Space
}

\author{
M. Radin \\ Department of Physics, K. N. Toosi University of Technology, P.O. Box 16315-1618, Tehran, Iran \\ Correspondence should be addressed to M. Radin; radin@kntu.ac.ir
}

Received 28 August 2016; Revised 27 October 2016; Accepted 29 November 2016; Published 21 February 2017

Academic Editor: Mohammadreza Hadizadeh

Copyright ( 2017 M. Radin. This is an open access article distributed under the Creative Commons Attribution License, which permits unrestricted use, distribution, and reproduction in any medium, provided the original work is properly cited. The publication of this article was funded by SCOAP .

In this paper we have solved the nonrelativistic form of the Lippmann-Schwinger equation in the momentum-helicity space by inserting a spin-dependent quark-antiquark potential model numerically. To this end, we have used the momentum-helicity basis states for describing a nonrelativistic reduction of one-gluon exchange potential. Then we have calculated the mass spectrum of the charmonium $\psi(c \bar{c})$, and finally we have compared the results with the other theoretical results and experimental data.

\section{Introduction}

During the past years, several models and methodological approaches based on solving the relativistic and nonrelativistic form of the Schrödinger or Lippmann-Schwinger equation have been developed for studying the light and heavy mesons in the coordinate and momentum spaces, respectively.

Recently, the three-dimensional approach based on momentum-helicity basis states for studding the nucleonnucleon scattering and deuteron state has been developed $[1,2]$. We extend this approach to particle physics problems by solving the nonrelativistic form of the Lippmann-Schwinger equation to obtain the mass spectrum of the heavy mesons using the nonrelativistic quark-antiquark interaction in terms of a linear confinement, a Coulomb, and various spindependent pieces.

In the heavy-quark $(c, b)$ mesons the differences between energy levels are small compared to the particle masses. Hence, the nonrelativistic Lippmann-Schwinger equation can be used to study their quantum behavior. To this end, we have used the nonrelativistic form of the LippmannSchwinger equation in the momentum-helicity representation to study the charmonium as a heavy meson. For this purpose, we have used a nonrelativistic quark-antiquark potential based on one-gluon exchange in the momentumhelicity representation.
This article is organized as follows. In Section 2, the nonrelativistic Lippmann-Schwinger equation in the momentum-helicity basis states which leads to coupled and uncoupled integral equations for various quantum numbers is presented briefly. In Section 3, a spin-dependent quarkantiquark potential model is described in the momentumhelicity basis states. The details of the numerical calculations and the results obtained for the charmonium are presented in Section 4. Finally, a summary and an outlook are provided in Section 5.

\section{Lippmann-Schwinger Equation in Momentum-Helicity Basis States}

The nonrelativistic form of the homogenous LippmannSchwinger equation for describing the heavy meson bound state is given by

$$
\left|\Phi_{j}^{M_{j}}\right\rangle=\frac{1}{E-p^{2} / m} V\left|\Phi_{j}^{M_{j}}\right\rangle,
$$

where $V$ denotes the quark-antiquark interaction, $m$ is mass of the quark or antiquark, and $\left|\Phi_{j}^{M_{j}}\right\rangle$ is the meson bound state with the total angular momentum $j . M_{j}$ is projection of the total angular momentum $j$ along the quantization axis. The 
integral form of this equation in the momentum-helicity basis states is written as [3]

$$
\begin{aligned}
& \Phi_{S j}^{M_{j}}(p) \\
& \quad=\frac{2 \pi}{E-p^{2} / m} \sum_{\Lambda^{\prime}} \int_{0}^{\infty} d p^{\prime} p^{\prime 2} V_{M_{j} \Lambda^{\prime}}^{S}\left(p, p^{\prime}\right) \Phi_{S j}^{\Lambda^{\prime}}\left(p^{\prime}\right),
\end{aligned}
$$

with

$$
\begin{aligned}
& V_{M_{j} \Lambda^{\prime}}^{S}\left(p, p^{\prime}\right) \\
& \quad=\int_{-1}^{1} d \cos \theta^{\prime} V_{M_{j} \Lambda^{\prime}}^{S}\left(p, p^{\prime}, \theta^{\prime}\right) d_{M_{j} \Lambda^{\prime}}^{j}\left(\theta^{\prime}\right)
\end{aligned}
$$

where $p$ is the magnitude of the relative momentum of the quark and antiquark, $S$ is the total spin of meson, $\Lambda$ is the spin projection along the relative momentum, and $d_{M_{j} \Lambda^{\prime}}^{j}\left(\theta^{\prime}\right)$ are the rotation matrices. For an arbitrary total angular momentum $j$, and singlet case of the total spin state, (2) leads to one equation:

$$
\begin{aligned}
& \Phi_{0 j}^{M_{j}}(p) \\
& \quad=\frac{2 \pi}{E-p^{2} / m} \int_{0}^{\infty} d p^{\prime} p^{\prime 2} V_{M_{j} 0}^{0}\left(p, p^{\prime}\right) \Phi_{0 j}^{0}\left(p^{\prime}\right) .
\end{aligned}
$$

Also for $j=0$ and triplet case of the total spin state, (2) leads to one equation as follows:

$$
\Phi_{1 j}^{0}(p)=\frac{2 \pi}{E-p^{2} / m} \int_{0}^{\infty} d p^{\prime} p^{\prime 2} V_{00}^{1}\left(p, p^{\prime}\right) \Phi_{1 j}^{0}\left(p^{\prime}\right) .
$$

For $S=1$ and $j>0$ it is more complicated. For example, for $j=1$, (2) leads to one equation for channel $P$ and two coupled equations for channels $S$ and $D$ as follows:

$$
\begin{aligned}
& \Psi_{111}(p)=\frac{2 \pi}{E-p^{2} / m} \int_{0}^{\infty} d p^{\prime} p^{\prime 2}\left[V_{11}^{1}\left(p, p^{\prime}\right)\right. \\
& \left.-V_{-11}^{1}\left(p, p^{\prime}\right)\right] \Psi_{111}\left(p^{\prime}\right), \\
& \Psi_{011}(p)=\frac{2 \pi}{E-p^{2} / m} \frac{1}{3} \int_{0}^{\infty} d p^{\prime} p^{\prime 2}\left\{\left[2 V_{11}^{1}\left(p, p^{\prime}\right)\right.\right. \\
& +2 V_{01}^{1}\left(p, p^{\prime}\right)+V_{00}^{1}\left(p, p^{\prime}\right)+2 V_{10}^{1}\left(p, p^{\prime}\right) \\
& \left.+2 V_{-11}^{1}\left(p, p^{\prime}\right)\right] \Psi_{011}\left(p^{\prime}\right)+\sqrt{2}\left[V_{11}^{1}\left(p, p^{\prime}\right)\right. \\
& +V_{01}^{1}\left(p, p^{\prime}\right)-V_{00}^{1}\left(p, p^{\prime}\right)-2 V_{10}^{1}\left(p, p^{\prime}\right) \\
& \left.\left.+V_{-11}^{1}\left(p, p^{\prime}\right)\right]\right\} \Psi_{211}\left(p^{\prime}\right), \\
& \Psi_{211}(p)=\frac{2 \pi}{E-p^{2} / m} \frac{1}{3} \int_{0}^{\infty} d p^{\prime} p^{\prime 2}\left\{\sqrt { 2 } \left[V_{11}^{1}\left(p, p^{\prime}\right)\right.\right. \\
& \quad-2 V_{01}^{1}\left(p, p^{\prime}\right)-V_{00}^{1}\left(p, p^{\prime}\right)+V_{10}^{1}\left(p, p^{\prime}\right) \\
& \left.+V_{-11}^{1}\left(p, p^{\prime}\right)\right] \Psi_{011}\left(p^{\prime}\right)+\left[V_{11}^{1}\left(p, p^{\prime}\right)\right.
\end{aligned}
$$

$$
\begin{aligned}
& -2 V_{01}^{1}\left(p, p^{\prime}\right)+2 V_{00}^{1}\left(p, p^{\prime}\right)-2 V_{10}^{1}\left(p, p^{\prime}\right) \\
& \left.\left.+V_{-11}^{1}\left(p, p^{\prime}\right)\right]\right\} \Psi_{211}\left(p^{\prime}\right)
\end{aligned}
$$

where $\Psi_{l S j}(p)$ is the partial wave component of the wave function which is connected to the momentum-helicity component of the wave function as [3]

$$
\Phi_{j S}^{\Lambda}(p)=\sum_{l} \sqrt{\frac{2 l+1}{4 \pi}} C(l S j ; 0 \Lambda \Lambda) \Psi_{l S j}(p) .
$$

The inverse relation is written as

$$
\Psi_{l S j}(p)=\frac{\sqrt{4 \pi(2 l+1)}}{2 j+1} \sum_{\Lambda} C(l S j ; 0 \Lambda \Lambda) \Phi_{j S}^{\Lambda}(p) .
$$

\section{Quark-Antiquark Potential in Momentum- Helicity Basis States}

The spin-dependent potential model that we have used in our calculations is sum of the Linear and a simple nonrelativistic reduction of an effective one-gluon exchange potential without retardation. This potential in the coordinate space is given in terms of [4]

$$
\begin{gathered}
V(\mathbf{r}, \mathbf{p})=\sigma r+f_{c} \alpha_{s}\left\{\frac{1}{r}-\frac{\pi}{m^{2}} \delta(\mathbf{r})+\frac{1}{m^{2}} \frac{\mathbf{p} \cdot \mathbf{p}}{r}\right. \\
-\frac{3}{4 m^{2}} \frac{\mathbf{L} \cdot\left(\boldsymbol{\sigma}_{1}+\boldsymbol{\sigma}_{2}\right)}{r^{3}}-\frac{2 \pi}{3 m^{2}} \delta(\mathbf{r})\left(\boldsymbol{\sigma}_{1} \cdot \boldsymbol{\sigma}_{2}\right) \\
\left.-\frac{1}{4 m^{2}} \frac{3\left(\boldsymbol{\sigma}_{1} \cdot \widehat{\mathbf{r}}\right)\left(\boldsymbol{\sigma}_{1} \cdot \widehat{\mathbf{r}}\right)-\left(\boldsymbol{\sigma}_{1} \cdot \boldsymbol{\sigma}_{2}\right)}{r^{3}}\right\},
\end{gathered}
$$

where $\sigma$ is the string tension, $\alpha_{s}$ is the strong-interaction fine-structure constant, $f_{c}$ is the color factor which is $-4 / 3$ for quark-antiquark and $-2 / 3$ for quark-quark, $\sigma_{1}$ and $\sigma_{2}$ are the Pauli matrices, and $\mathbf{L}$ is the total orbital angular momentum operator. Fourier transformation of this potential to momentum space yields

$$
\begin{aligned}
& \left\langle\mathbf{p}|V| \mathbf{p}^{\prime}\right\rangle=\sigma\left[\delta(\mathbf{q}) r_{c}\right. \\
& \left.+\frac{1}{2 \pi^{2} q^{4}}\left(2 \cos \left(q r_{c}\right)-2+q r_{c} \sin \left(q r_{c}\right)\right)\right] \\
& +f_{c} \alpha_{s} e^{-\lambda^{2} q^{2}}\left\{\left[\frac{\delta(\mathbf{q})}{r_{c}}+\frac{1}{2 \pi^{2} q^{2}}\left(1-\frac{\sin \left(q r_{c}\right)}{q r_{c}}\right)\right]\right. \\
& +\left(1+\frac{p^{2}}{m^{2}}\right)-\frac{1}{8 \pi^{2} m^{2}}+\frac{3}{8 \pi^{2} m^{2} q^{2}} i\left(\boldsymbol{\sigma}_{1}+\boldsymbol{\sigma}_{2}\right) \cdot \mathbf{p} \\
& \quad \times \mathbf{p}^{\prime}-\frac{1}{12 \pi^{2} m^{2}}\left(\boldsymbol{\sigma}_{1} \cdot \boldsymbol{\sigma}_{2}\right) \\
& \left.+\frac{1}{24 \pi^{2} m^{2}}\left[3\left(\boldsymbol{\sigma}_{1} \cdot \widehat{\mathbf{q}}\right)\left(\boldsymbol{\sigma}_{2} \cdot \widehat{\mathbf{q}}\right)-\left(\boldsymbol{\sigma}_{1} \cdot \boldsymbol{\sigma}_{2}\right)\right]\right\}
\end{aligned}
$$


where $\mathbf{q}=\mathbf{p}^{\prime}-\mathbf{p}$ is the momentum transfer. The kernels of integral equations have singularity. To overcome this problem we have used the regularized form of linear confining and Coulomb parts of the potential [5]. Details of Fourier transformation of regularized parts of the potential are given in the appendix. Also we have used a Gaussian form factor, $\exp \left((-1 / 2) \lambda^{2} q^{2}\right)$ at the quark-gluon vertex as in [6] to remove singularity of the kernels due to existence of one-gluon exchange potential. The variable $\lambda$ can be interpreted as size of the quark. In [7] the point-like quark-gluon vertex is replaced by a form factor, $1 /\left(q^{2}+\beta^{2}\right)$ in which $\beta^{-1}$ is the effective quark size to eliminate the singularity. In this work we have used both regularized form and Gaussian form factor for Coulomb and $f_{c} \alpha_{s} \mathbf{p}^{2} /\left(\mathrm{m}^{2} r\right)$ parts of the potential which cause the convergence of numerical results faster. Therefore, the final form of the potential in the momentum-helicity space is written as

$$
\begin{aligned}
& V_{\Lambda \Lambda^{\prime}}^{S}\left(\mathbf{p}, \mathbf{p}^{\prime}\right) \equiv\left\langle\mathbf{p} S \Lambda|V| \mathbf{p}^{\prime} S \Lambda^{\prime}\right\rangle=\sigma\left\langle\widehat{\mathbf{p}} S \Lambda \mid \hat{\mathbf{p}}^{\prime} S \Lambda^{\prime}\right\rangle \\
& \cdot\left[\delta(\mathbf{q}) r_{c}+\frac{1}{2 \pi^{2} q^{4}}\left(2 \cos \left(q r_{c}\right)-2\right.\right. \\
& \left.\left.+q r_{c} \sin \left(q r_{c}\right)\right)\right]+f_{c} \alpha_{s} e^{-\lambda^{2} q^{2}}\left\langle\widehat{\mathbf{p}} S \Lambda \mid \widehat{\mathbf{p}}^{\prime} S \Lambda^{\prime}\right\rangle\left\{\frac{1}{r_{c}}\right. \\
& \cdot \delta(\mathbf{q})+\frac{1}{2 \pi^{2} q^{2}}\left(1-\frac{\sin \left(q r_{c}\right)}{q r_{c}}\right)\left(1+\frac{p^{2}}{m^{2}}\right) \\
& -\frac{1}{8 \pi^{2} m^{2}}-\frac{1}{12 \pi^{2} m^{2}}(2 S(S+1)-3)+\frac{3}{8 \pi^{2} m^{2}} \\
& \cdot \frac{p p^{\prime}}{q^{2}}\left[\gamma S(S+1)-2 \Lambda \Lambda^{\prime}\right. \\
& \left.-\frac{1}{\gamma}\left(S(S+1)-2 \Lambda^{\prime 2}-2 \Lambda^{2}-2 \Lambda^{\prime 2} \Lambda^{2}\right)\right] \\
& -\frac{1}{24 \pi^{2} m^{2} q^{2}}\left[6 p p^{\prime} \Lambda \Lambda^{\prime}+2 p^{\prime 2}\left(S(S+1)-3 \Lambda^{\prime 2}\right)\right. \\
& +2 p^{2}\left(S(S+1)-3 \Lambda^{2}\right)-p p^{\prime} \gamma S(S+1) \\
& \left.\left.-3 \frac{p p^{\prime}}{\gamma}\left(S(S+1)-2 \Lambda^{\prime 2}-2 \Lambda^{2}-2 \Lambda^{\prime 2} \Lambda^{2}\right)\right]\right\},
\end{aligned}
$$

where $\gamma=\widehat{\mathbf{p}}^{\prime} \cdot \widehat{\mathbf{p}}=\cos \theta \cos \theta^{\prime}+\sin \theta \sin \theta^{\prime} \cos \left(\varphi-\varphi^{\prime}\right)$ and $|\mathbf{p} ; \widehat{\mathbf{p}} S \Lambda\rangle$ is the momentum-helicity basis state which is eigenstate of the helicity operator $\mathbf{S} \cdot \widehat{\mathbf{p}}$ as

$$
\mathbf{S} \cdot \widehat{\mathbf{p}}|\mathbf{p} ; \widehat{\mathbf{p}} S \Lambda\rangle=\Lambda|\mathbf{p} ; \widehat{\mathbf{p}} S \Lambda\rangle .
$$

Also we have [1]

$$
\left\langle\widehat{\mathbf{p}} S \Lambda \mid \widehat{\mathbf{p}}^{\prime} S \Lambda^{\prime}\right\rangle=\sum_{N=-S}^{S} e^{i N\left(\varphi-\varphi^{\prime}\right)} d_{N \Lambda}^{S}(\theta) d_{N \Lambda^{\prime}}^{S}\left(\theta^{\prime}\right) .
$$

If the vector $\mathbf{p}$ is along $z$-direction, it is clear that (15) is reduced to

$$
\left\langle\widehat{\mathbf{z}} S \Lambda \mid \widehat{\mathbf{p}}^{\prime} S \Lambda^{\prime}\right\rangle=e^{-i \Lambda \varphi^{\prime}} d_{\Lambda \Lambda^{\prime}}^{S}\left(\theta^{\prime}\right) .
$$

TABLE 1: Parameters of the model.

\begin{tabular}{lc}
\hline$\sigma[\mathrm{GeV} / \mathrm{fm}]$ & 1.222 \\
$\lambda\left[\mathrm{GeV}^{-1}\right]$ & 0.3154 \\
$m[\mathrm{GeV}]$ & 1.269 \\
$\alpha_{s}$ & 0.2863 \\
$r_{c}[\mathrm{fm}]$ & 10 \\
\hline
\end{tabular}

For numerical calculations we need the matrix elements of the potential $V_{\Lambda \Lambda^{\prime}}^{S}\left(p, p^{\prime}, \theta^{\prime}\right)$. These matrix elements are related to the matrix elements of (13) as follows:

$$
V_{\Lambda \Lambda^{\prime}}^{S}\left(p, p^{\prime}, \theta^{\prime}\right)=e^{i \Lambda \varphi^{\prime}}\left\langle p \mathbf{z} ; \widehat{\mathbf{z}} S \Lambda|V| \mathbf{p}^{\prime} ; \widehat{\mathbf{p}}^{\prime} S \Lambda^{\prime}\right\rangle
$$

By considering (13), (16), and (17), the final form of the matrix elements of the potential which is inserted in the numerical calculations is written as

$$
\begin{aligned}
V_{\Lambda \Lambda^{\prime}}^{S}\left(p, p^{\prime}, \theta^{\prime}\right)=\sigma d_{\Lambda \Lambda^{\prime}}^{S}\left(\theta^{\prime}\right)\left[\delta(\mathbf{q}) r_{c}\right. \\
\left.+\frac{1}{2 \pi^{2} q^{4}}\left(2 \cos \left(q r_{c}\right)-2+q r_{c} \sin \left(q r_{c}\right)\right)\right] \\
+f_{c} \alpha_{s} e^{-\lambda^{2} q^{2}} d_{\Lambda \Lambda^{\prime}}^{S}\left(\theta^{\prime}\right)\left\{\frac{1}{r_{c}} \delta(\mathbf{q})+\frac{1}{2 \pi^{2} q^{2}}(1\right. \\
\left.-\frac{\sin \left(q r_{c}\right)}{q r_{c}}\right)\left(1+\frac{p^{2}}{m^{2}}\right)-\frac{1}{8 \pi^{2} m^{2}} \\
-\frac{1}{12 \pi^{2} m^{2}}(2 S(S+1)-3)+\frac{3}{8 \pi^{2} m^{2}} \\
+\frac{p p^{\prime}}{q^{2}}\left[\gamma S(S+1)-2 \Lambda \Lambda^{\prime}\right. \\
\left.-\frac{1}{\gamma}\left(S(S+1)-2 \Lambda^{\prime 2}-2 \Lambda^{2}-2 \Lambda^{\prime 2} \Lambda^{2}\right)\right] \\
-\frac{1}{24 \pi^{2} m^{2} q^{2}}\left[6 p p^{\prime} \Lambda \Lambda^{\prime}+2 p^{\prime 2}\left(S(S+1)-3 \Lambda^{\prime 2}\right)\right. \\
+2 p^{2}\left(S(S+1)-3 \Lambda^{2}\right)-p p^{\prime} \gamma S(S+1) \\
\left.\left.-3 \frac{p p^{\prime}}{\gamma}\left(S(S+1)-2 \Lambda^{\prime 2}-2 \Lambda^{2}-2 \Lambda^{\prime 2} \Lambda^{2}\right)\right]\right\},
\end{aligned}
$$

with $\gamma=\widehat{\mathbf{p}}^{\prime} \cdot \widehat{\mathbf{z}}=\cos \theta^{\prime}$.

\section{Discussion and Numerical Results}

For numerical calculations as a first step we have used the Gaussian quadrature grid points to discretize the momentum and the angle variables. The integration interval for the momentum is covered by two different hyperbolic and linear mappings of the Gauss-Legendre points from the interval 
TABLE 2: Comparison of the obtained charmonium mass spectrum with the experimental data and another work.

\begin{tabular}{lcccc}
\hline$n^{2 S+1} L_{J}$ & Candidate & Exp. [8] & Ref. [9] & Mass [MeV] \\
\hline $1^{1} S_{0}$ & $\eta_{c}$ & $2980.4 \pm 1.2$ & 2980 & 3097 \\
$1^{3} S_{1}$ & $J / \psi$ & $3096.916 \pm 0.011$ & 3527 & 3096.9 \\
$1^{1} P_{1}$ & $h_{c}$ & $3526.21 \pm 0.25$ & 3430 & 3526.2 \\
$1^{3} P_{0}$ & $\chi_{c 0}$ & $3415.16 \pm 0.35$ & 3503 & 3397.4 \\
$1^{3} P_{1}$ & $\chi_{c 1}$ & $3510.59 \pm 0.10$ & 3674 & 3503.5 \\
$2^{1} S_{0}$ & $\eta_{c}^{\prime}$ & $3638 \pm 5$ & 3765 & 3760.8 \\
$2^{3} S_{1}$ & $\psi^{\prime}$ & $3686.093 \pm 0.034$ & 3855 & 3850.6 \\
$1^{3} D_{1}$ & $\psi^{\prime \prime}$ & $3770 \pm 2.4$ & 4291 & 4285.4 \\
$3^{3} S_{1}$ & $\psi^{\prime \prime \prime}$ & $4040 \pm 10$ & & \\
\hline
\end{tabular}

TABLE 3: Percent of each partial wave in mixed charmonium states.

\begin{tabular}{lccc}
\hline$n^{2 S+1} L_{J}$ & $c \bar{c}$ & $P_{S} \%$ & $P_{D} \%$ \\
\hline $1^{3} S_{1}\left(1^{3} S_{1}-1^{3} D_{1}\right)$ & $J / \psi$ & 99.93 & 0.07 \\
$2^{3} S_{1}\left(2^{3} S_{1}-2^{3} D_{1}\right)$ & $\psi^{\prime}$ & 99.90 & 0.10 \\
$3^{3} S_{1}\left(3^{3} S_{1}-3^{3} D_{1}\right)$ & $\psi^{\prime \prime \prime}$ & 99.88 & 0.12 \\
$1^{3} D_{1}\left(1^{3} D_{1}-1^{3} S_{1}\right)$ & $\psi^{\prime \prime}$ & 99.88 & 0.12 \\
\hline
\end{tabular}

TABLE 4: The calculated charmonium mass spectrum as function of the number of grid points $N_{P 1}, N_{P 1}$, and $N_{\theta}$.

\begin{tabular}{cccccccccccc}
\hline$N_{p 1}$ & $N_{p 2}$ & $N_{\theta}$ & $\eta_{c}$ & $J / \psi$ & $h_{c}$ & $\chi_{c 0}$ & $\chi_{c 1}$ & $\eta_{c}^{\prime}$ & $\psi^{\prime}$ & $\psi^{\prime \prime}$ & $\psi^{\prime \prime \prime}$ \\
\hline 100 & 100 & 140 & 2980.602 & 3096.942 & 3526.244 & 3397.517 & 3503.552 & 368.3357 & 3760.778 & 3850.573 & 4285.415 \\
100 & 100 & 160 & 2980.420 & 3096.951 & 3526.226 & 3397.449 & 3503.541 & 3683.153 & 3760.782 & 3850.563 & 4285.410 \\
100 & 100 & 180 & 2980.370 & 3096.954 & 3526.221 & 3397.434 & 3503.539 & 3683.095 & 3760.784 & 3850.561 & 4285.409 \\
100 & 100 & 200 & 2980.356 & 3096.954 & 3526.219 & 3397.431 & 3503.538 & 3683.080 & 3760.784 & 3850.560 & 4285.409 \\
100 & 100 & 220 & 2980.353 & 3096.954 & 3526.219 & 3397.430 & 3503.538 & 3683.075 & 3760.784 & 3850.560 & 4285.409 \\
100 & 100 & 240 & 2980.352 & 3.096954 & 3526.219 & 3397.430 & 3503.538 & 3683.074 & 3760.784 & 3850.560 & 4285.409 \\
100 & 100 & 260 & 2980.352 & 3.096954 & 3526.219 & 3397.430 & 3503.538 & 3683.074 & 3760.784 & 3850.560 & 4285.409 \\
\hline 100 & 60 & 200 & 2975.716 & 3097.131 & 3525.746 & 3397.449 & 3394.900 & 3503.182 & 3677.378 & 3850.232 & 4284.965 \\
100 & 80 & 200 & 2980.218 & 3096.961 & 3526.205 & 3397.376 & 3503.530 & 3682.920 & 3760.787 & 3850.553 & 4285.405 \\
100 & 100 & 200 & 2980.356 & 3096.954 & 3526.219 & 3397.431 & 3503.541 & 3683.538 & 3760.080 & 3850.560 & 4285.409 \\
100 & 120 & 200 & 2980.356 & 3096.954 & 3526.219 & 3397.431 & 3503.541 & 3503.538 & 3683.080 & 3850.560 & 4285.409 \\
\hline 50 & 100 & 200 & 2980.356 & 3096.954 & 3526.219 & 3397.429 & 3503.538 & 3683.080 & 3760.784 & 3850.558 & 4285.409 \\
80 & 100 & 200 & 2980.356 & 3096.954 & 3526.219 & 3397.430 & 3503.538 & 3683.080 & 3760.784 & 3850.560 & 4285.409 \\
100 & 100 & 200 & 2980.356 & 3096.954 & 3526.219 & 3397.431 & 3503.541 & 3683.080 & 3760.784 & 3850.560 & 4285.409 \\
\hline
\end{tabular}

$[-1,+1]$ to the intervals $\left[0, p_{2}\right] \cup\left[p_{2}, p_{\max }\right]$, respectively, as follows:

$$
\begin{aligned}
& p=\frac{1+x}{1 / p_{1}+\left(2 / p_{2}-1 / p_{1}\right) x}, \\
& p=\frac{p_{\max }-p_{2}}{2} x+\frac{p_{\max }+p_{2}}{2} .
\end{aligned}
$$

Then we have calculated the matrix elements of the potential $V_{\Lambda \Lambda^{\prime}}\left(p, p^{\prime}, \theta^{\prime}\right)$, from (18). According to (3) integration over the spherical angle variable $\theta^{\prime}$ has been done independently. Finally, we have solved the integral equations (4)-(8) as eigenvalue equations. The integration over momentum variable is cut off at $q_{\max }=10 \mathrm{GeV}$. This selection is carried out so that the numerical results do not depend on this choice. The typical values for $p_{1}$ and $p_{2}$ are $1 \mathrm{GeV}$ and $3 \mathrm{GeV}$, respectively. These selections are done till the total number of grid points for momentum intervals is decreased. Other selections can be done but by different grid points for momentum variables.

The parameters of the potential model which are shown in Table 1 are fixed by a fit to the masses of the states $\eta_{c}, J / \psi$, and $h_{c}$, similar to what is done in [9]. The results of charmonium mass spectrum are shown in Table 2 . They are compared with the experimental data and another theoretical work. As it is clear from (7) and (8), the existence of the tensor term in the potential causes the coupling of the $S$ - and $D$-partial waves and this coupling as is shown in Table 3 is so weak. I show the mixed charmonium states in Table 2 by their dominant partial wave.

As a test of our numerical calculations we have shown convergence of the results as a function of number of grid points $N_{P 1}, N_{P 2}$, and $N_{\theta}$ for the momentum and angle 
variables in Table 4. $N_{P 1}$ and $N_{P 2}$ are the number of grid points for the intervals $\left[0, p_{2}\right]$ and $\left[p_{2}, p_{\max }\right]$, respectively. $N_{\theta}$ is corresponding to number of grid points for spherical angle variable. In our calculations we have chosen $N_{P 1}=100$, $N_{P 2}=100$ and $N_{\theta}=200$ grid points for to achieve an acceptable accuracy.

\section{Summary and Outlook}

In this paper we have extended an approach based on momentum-helicity basis states for calculation of mass spectrum of heavy mesons by solving nonrelativistic form of the Lippmann-Schwinger equation. As an application we have used this approach to obtain the mass spectrum of charmonium. The advantage of working with helicity states is that states are the eigenstates of the helicity operator appearing in the quark-antiquark potential. Thus, using the helicity representation is less complicated than using the spin representation with a fixed quantization axis for representation of spin-dependent potentials. This work is the first step toward studying single, double, and triple heavy-flavor baryons in the framework of the nonrelativistic quark model by formulation of the Faddeev equation in the 3D momentum-helicity representation. Furthermore, we can apply this formalism straightforwardly for investigation of heavy pentaquark systems, which can be considered as two-body (heavy meson and baryon) systems with mesonnucleon potentials which is underway.

\section{Appendix}

\section{Fourier Transformation of the Regularized Linear Confining and Coulomb Parts of the Potential}

The three-dimensional Fourier transformation of the potential $V(r)$ is defined as

$$
V\left(\mathbf{p}, \mathbf{p}^{\prime}\right)=\frac{1}{2 \pi^{2} q^{2}} \int_{0}^{\infty} d r r V(r) \sin q r
$$

where $\mathbf{q}=\left|\mathbf{p}-\mathbf{p}^{\prime}\right|$. Fourier transformation of the regularized linear confining and Coulomb parts of the quark-antiquark potential is written as

$$
\begin{aligned}
& V\left(\mathbf{p}, \mathbf{p}^{\prime}\right)=\frac{1}{2 \pi^{2} q^{2}}\left\{\int_{0}^{r_{c}} d r r V(r) \sin q r\right. \\
& \left.+V\left(r_{c}\right) \int_{r_{c}}^{\infty} d r r \sin q r\right\} \\
& =\frac{1}{2 \pi^{2} q^{2}}\left\{\int_{0}^{r_{c}} d r r V(r) \sin q r\right. \\
& \left.+V\left(r_{c}\right) \int_{0}^{\infty} d r r \sin q r-V\left(r_{c}\right) \int_{0}^{r_{c}} d r r \sin q r\right\}
\end{aligned}
$$

$$
\begin{aligned}
& =\frac{1}{2 \pi^{2} q^{2}}\left\{\int_{0}^{r_{c}} d r r V(r) \sin q r+V\left(r_{c}\right) \delta(\mathbf{q})\right. \\
& \left.-V\left(r_{c}\right) \int_{0}^{r_{c}} d r r \sin q r\right\},
\end{aligned}
$$

where potential is kept fixed at cutoff $r_{c}$. Therefore inserting the linear $V(r)=\sigma r$ and Coulomb $V(r)=f_{c} \alpha_{s} / r$, parts of quark-antiquark potential, in the above equation and calculation of corresponding integrals analytically yield

$$
\begin{aligned}
& V\left(\mathbf{p}, \mathbf{p}^{\prime}\right)=\sigma\left[\delta(\mathbf{q}) r_{c}\right. \\
& \left.+\frac{1}{2 \pi^{2} q^{4}}\left(2 \cos \left(q r_{c}\right)-2+q r_{c} \sin \left(q r_{c}\right)\right)\right], \\
& V\left(\mathbf{p}, \mathbf{p}^{\prime}\right)=f_{c} \alpha_{s}\left[\frac{\delta(\mathbf{q})}{r_{c}}+\frac{1}{2 \pi^{2} q^{2}}\left(1-\frac{\sin \left(q r_{c}\right)}{q r_{c}}\right)\right] .
\end{aligned}
$$

\section{Competing Interests}

The author declares that there is no conflict of interests regarding the publication of this paper.

\section{References}

[1] I. Fachruddin, C. Elster, and W. Glöckle, "Nucleon-nucleon scattering in a three dimensional approach," Physical Review C, vol. 62, no. 4, Article ID 044002, 2000.

[2] I. Fachruddin, C. Elster, and W. Glöckle, "New forms of deuteron equations and wave function representations," Physical Review C-Nuclear Physics, vol. 63, no. 5, Article ID 054003, 2001.

[3] M. Radin and N. Tazimi, "Quark-antiquark bound state in momentum-helicity representation," Physical Review D, vol. 90, no. 8, Article ID 085020, 2014.

[4] S. N. Gupta, S. F. Radford, and W. W. Repko, "Quarkonium spectra and quantum chromodynamics," Physical Review D, vol. 26, no. 11, pp. 3305-3308, 1982.

[5] M. R. Hadizadeh and L. Tomio, "Solution of two-body bound state problems with confining potentials," in Proceedings of the International Conference "1st Stars and Galaxies: Challenges for the Next Decade”, pp. 334-337, AIP, São Paulo, Brazil, March 2010.

[6] J. Carlson, J. B. Kogut, and V. R. Pandharipande, "Hadron spectroscopy in a flux-tube quark model," Physical Review D, vol. 28, no. 11, article 2807, 1983.

[7] D. P. Stanley and D. Robson, "Nonperturbative potential model for light and heavy quark-antiquark systems," Physical Review D, vol. 21, no. 11, pp. 3180-3196, 1980.

[8] S. Eidelman, K. G. Hayes, K. A. Olive et al., "Review of particle physics," Physics Letters B, vol. 592, no. 1-4, pp. 1-5, 2004.

[9] J. Eiglsperger, Quarkonium spectroscopy: beyond one-gluon exchange [Diploma thesis], Technische Universität München, Munich, Germany, 2007. 

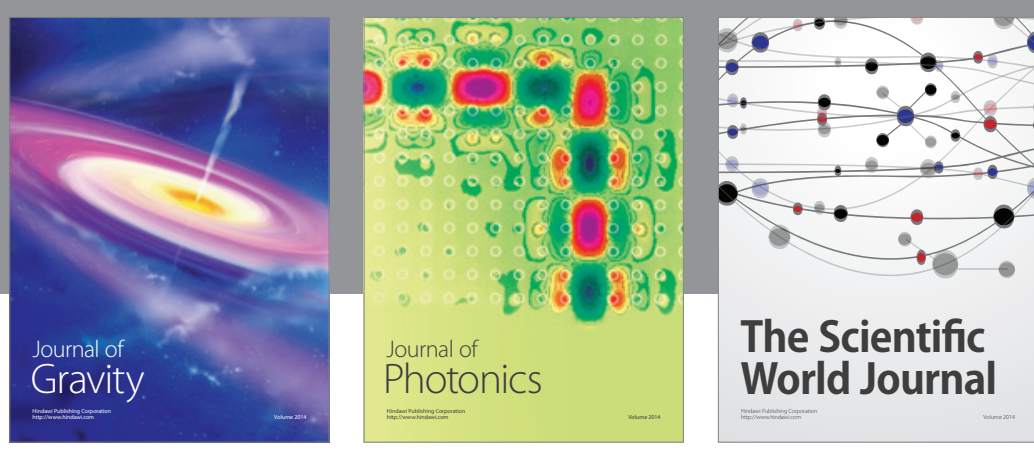

The Scientific World Journal
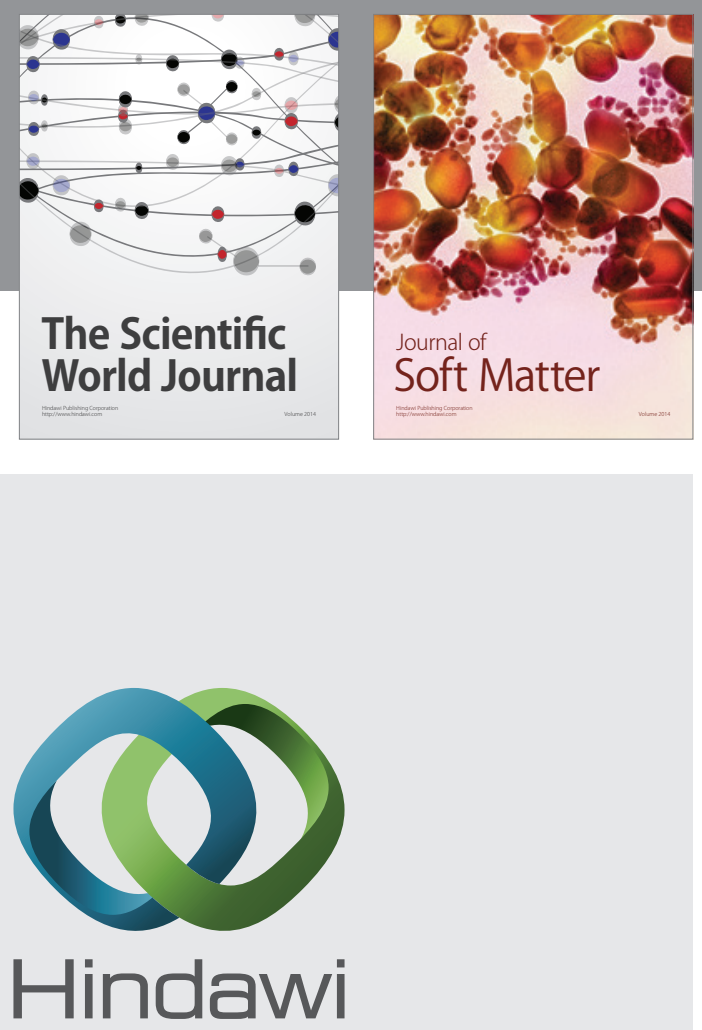

Submit your manuscripts at

https://www.hindawi.com
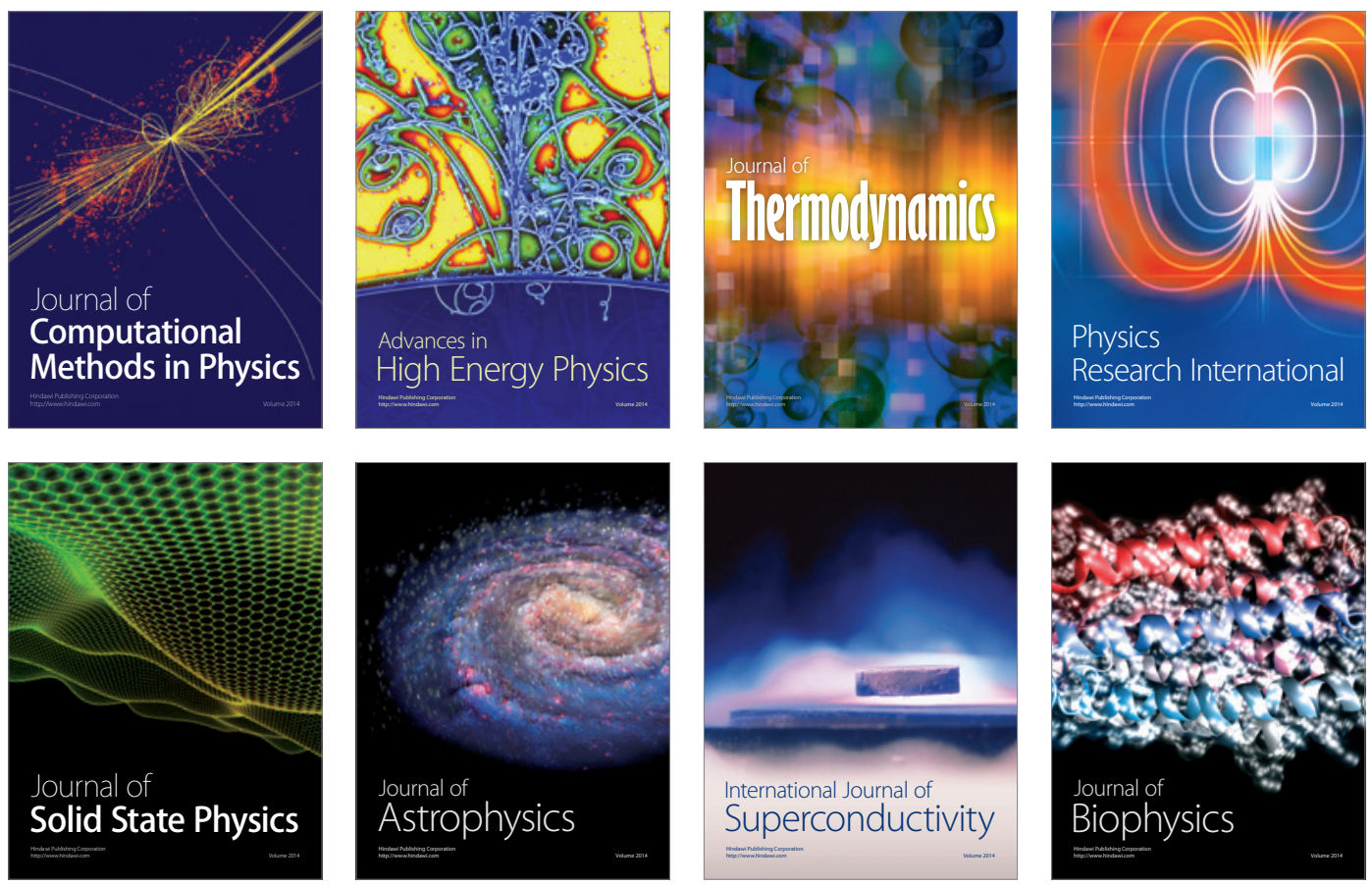
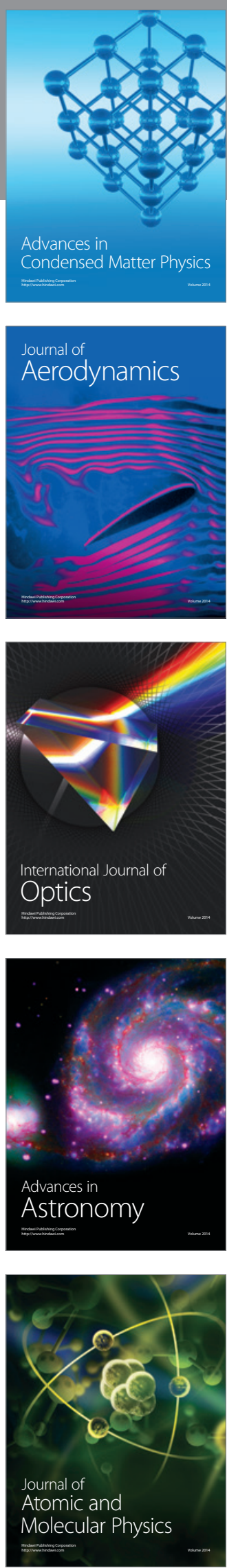